Las representaciones de los estudiantes de Psicología acerca de las prácticas de enseñanza. Páginas 303-312

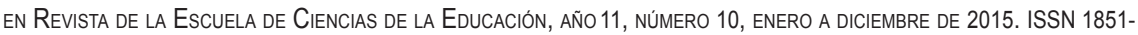
6297. ISSN EN LINEA 2362-3349.

\title{
LAS REPRESENTACIONES DE LOS ESTUDIANTES DE PSICOLOGÍA ACERCA DE LAS PRÁCTICAS DE ENSEÑANZA
}

\author{
Por Anahí Ayelen Marcovich* \\ Universidad Nacional del Comahue, Argentina. \\ anahi.marcovich@gmail.com
}

Recibido: 25/06/2015 Aceptado: 03/09/2015

\section{Resumen}

El presente artículo expone las conclusiones a las cuales se arribó en el marco de la elaboración de la tesis de Licenciatura en Ciencias de la Educación. La misma abordó las representaciones en torno a las prácticas de enseñanza de los estudiantes de Psicología de la FaCE-UNCo (Facultad de Ciencias de la Educación de la Universidad Nacional del Comahue). El interés que guió la indagación se articuló, por un lado, en preocupaciones personales que conducen a la reflexión sistemática acerca de las prácticas de enseñanza y al propósito de su mejora constante, y por el otro lado, se centró en desentrañar algunos de los múltiples sentidos que se ponen en juego en la situación pedagógica; considerando que las representaciones y significados de los estudiantes son ineludibles para comprender las formas que se acercan al conocimiento y su desempeño en las aulas, a la vez que se constituyen como punto de partida de su propio proceso de aprendizaje. En este sentido, atender a las representaciones que portan los estudiantes se presenta como una vía de conocimiento indispensable para aquellos que ejercemos la práctica docente, específicamente en la carrera de Psicología de reciente creación en Comahue.

\section{Palabras Clave:}

Representaciones - Estudiantes - Prácticas - Enseñanza - Psicología.

\footnotetext{
Prof. y Lic. en Ciencias de la Educación y Prof. en Enseñanza Primaria. Tesista de la Maestría en Psicología del Aprendizaje. Docente regular del Área: Desarrollo. Cátedra de Psicología de la Adolescencia, Profesorados de Historia y Profesorados de Letras, Geografía y Filosofía (Facultad de Humanidades, UNCo) y Asistente de Docencia cátedra Psicología del Desarrollo I, Psicología (Facultad de Ciencias de la Educación, UNCo).
} 
Revista de la Escuela de Ciencias de la Educación, año 11, número 10, enero a diciembre de 2015. Páginas 303-312. ISSN 1851-6297. ISSN EN LINEA 2362-3349. LAS REPRESENTACIONES DE LOS ESTUDIANTES DE PSICOLOGÍA ACERCA DE LAS prácticas de enseñanza. Anahí Ayelen Marcovich

\begin{abstract}
This paper presents the conclusions that were reached within the framework of the preparation of the thesis of Bachelor of Science in Education. It addressed the representations about the practices of teaching students of Psychology Face-UNCo (Faculty of Education at the National University of Comahue). The interest that guided the inquiry is articulated, on the one hand, on personal concerns that lead to the systematic reflection on teaching practices and the purpose of continuous improvement, and on the other hand, focused on unraveling some of the many senses that come into play in the teaching situation; considering that the representations and meanings of the students are unavoidable to understand the ways they approach the knowledge and performance in the classroom, while they are as a starting point for their own learning process. In this regard, take note of the representations that students carry is presented as a way for those who exercise essential teaching practice, specifically in the career of Psychology ups in Comahue knowledge.
\end{abstract}

Key words:

Representations - Practical - Teaching - Psychology - Students.

\title{
Las representaciones de los estudiantes de Psicología acerca de las prácticas de enseñanza
}

El presente trabajo presenta las conclusiones a las que se arribó en la elaboración de la tesis de Licenciatura en Ciencias de la Educación. Dicho trabajo tuvo como telón de fondo la inscripción de mi práctica profesional como docente en la carrera de Psicología en la Universidad Nacional del Comahue y en su Facultad de Ciencias de la Educación.

Fue a partir de la escucha atenta de las expresiones, de los estudiantes, acerca de las dificultades o facilidades que encontraban en la apropiación de contenidos, opiniones que aludían en forma recurrente a los atributos de los profesores y todo ello en una carrera donde mayoritariamente sus docentes no poseían formación pedagógica, lo que fue delineando el interés de indagación.

Por otra parte, los/las estudiantes de esta carrera manifestaban, sus expectativas respecto al desempeño profesional ligado a la práctica clínica en consultorio, a la práctica psicoanalítica específicamente. Dato que, se constataba, era simétrico en otras sedes académicas del país (Cattaneo \& Ruíz, 2007) lo cual animaba a pensar en cierta hegemonía del psicoanálisis inscriptas en la representación de las prácticas del psicólogo.

En este contexto fueron madurando interrogantes respecto de ¿cuáles eran las representaciones que tenían éstos estudiantes acerca de las prácticas de enseñanza?, si acaso ¿existía relación entre esas representaciones y la construcción del campo profesional? lo cual posibilitó la construcción del tema a indagar. 
Revista de la Escuela de Ciencias de la Educación, año 11, número 10, enero a diciembre de 2015. PÁginas 303-312. ISSN 1851-6297. ISSN EN LINEA 2362-3349. LAS REPRESENTACIONES DE LOS ESTUDIANTES DE PSICOLOGÍA ACERCA DE LAS prácticas de enseñanza. Anahi Ayelen Marcovich

En este sentido, describir e interpretar las representaciones de los estudiantes de psicología respecto de las prácticas de enseñanza, se constituyó en el objetivo general del proyecto de indagación, para luego y más específicamente identificar y describir sus representaciones, establecer la/s relación/es entre las representaciones identificadas y la construcción del campo profesional, reconocer y describir las características atribuidas a las prácticas de enseñanza relacionadas al campo laboral.

\section{Acerca de la perspectiva teórico-metodológica}

La búsqueda de antecedentes sobre la temática indagada condujo a la identificación de dos grandes categorías con las cuales establecer el diálogo constructivo de la tesis, lo cual a su vez se inscribió en el entrecruzamiento de dos campos disciplinares: Psicología y Didáctica.

En este sentido reconocer la categoría de representaciones, en términos psicosociales fue una de las definiciones a la hora de poder privilegiar eso que se quiere conocer con los sujetos que se quiere indagar. Entendiendo las representaciones como construcciones simbólicas que se crean y recrean en el curso de las interacciones sociales, constituyéndose en modos específicos de entender y comunicar la realidad, el marco de referencia obligado estuvo constituido por los aportes de Moscovici $(1979,1986)$ y Jodelet $(1986)$.

Moscovici (1986) alude -con el concepto de representaciones socialesal modo de pensar e interpretar la realidad cotidiana, como a las decisiones y acciones que se generan de la misma, y es por ello que el conocerlas nos dirá algo sobre la forma en que le damos significado a la realidad y a la vida diaria.

A este posicionamiento teórico se lo vinculó a la categoría de prácticas de enseñanza, posibilitando la construcción de un estado del arte que sostuvo la elección respecto de los referentes teóricos pertinentes en el contexto de la indagación diseñada.

Cabe destacar que si bien, el estudio de las prácticas de enseñanza nos remite a un viejo oficio que trasciende los niveles del sistema educativo, adquiere especificidad y singularidad en cada uno. Por ello y parafraseando a De Pascuale (2004), se entendió a las prácticas no como meras acciones o conjunto de técnicas que llevan a cabo los sujetos, sino como procesos públicos bajo los que determinadas actividades se constituyen y definen socialmente, se trata de prácticas que se constituyen continuamente de la forma en que los sujetos concretos entienden su sentido público y social.

Los aportes de Litwin, E. (1997, 1996) posibilitaron re conceptualizar aspectos de las mismas que adquirieron relevancia en el trabajo realizado, la diferenciación entre buena enseñanza y la enseñanza comprensiva, posibilitó la recuperación de la dimensión ética y epistemológica, desde su condición social, en los contextos y en el marco de las contradictorias relaciones entre 
Revista de la Escuela de Ciencias de la Educación, año 11, número 10, enero a diciembre de 2015. Páginas 303-312. ISSN 1851-6297. ISSN EN LINEA 2362-3349. LAS REPRESENTACIONES DE LOS ESTUDIANTES DE PSICOLOGÍA ACERCA DE LAS prácticas de enseñanza. Anahí Ayelen Marcovich

los actores en los ámbitos educativos. Por otra parte el adjetivo comprensivo refiere a visiones profundas acerca de la enseñanza, que propician la reflexión sobre las circunstancias de la vida del aula, los mecanismos que entran en juego como así también la complejidad de las relaciones y aprendizajes inherentes.

En un todo de acuerdo con los referentes teóricos, se optó por una indagación de tipo cualitativa, desde una perspectiva etnográfica. Este enfoque permitió conservar las acciones y el lenguaje original de los sujetos, posibilitando, penetrar en las estructuras de significación de las representaciones de los estudiantes. Dicho de otro modo, conocer los significados que tienen las prácticas de enseñanza para los estudiantes de psicología, conocer la diversidad de sus significaciones; es decir producir conocimiento a partir de las voces de sus protagonistas y no como resultado de una reflexión meramente especulativa. Este proceso, en el cual se amalgaman los datos en una relación dialéctica entre acción (praxis) y reflexión (teoría), en la búsqueda de la comprensión del objeto; fue combinado con un instrumento de enfoque cuantitativo, entendiendo que dicha articulación metodológica posibilitó una comprensión más amplia e integral del fenómeno que se abordó.

Se construyeron dos instrumentos para el trabajo de campo: cuestionario y entrevista en profundidad semi estructuradas. Estos instrumentos permitieron recuperar la singularidad textual e interpretativa de nuestros informantes claves (entrevistas) en un universo más amplio (encuestas). La decisión acerca de las fuentes de información que respaldarían la investigación se realizó desde el mismo momento en que se definió el diseño. Pues, tal como señala DıAncona (1999), al escoger una estrategia de investigación, implícita o explícitamente se está definiendo con qué métodos y tipo de información se va a trabajar. Por lo tanto, se está estableciendo cuáles son los aspectos del problema a investigar que serán abordados y de qué manera se intentará dar cuenta de los mismos. Esto significa que la decisión acerca del tipo de información con el cual operar tiene lugar desde el mismo momento en que comienza a delimitarse el objeto de estudio.

El análisis se llevó a cabo en dos niveles. Un primer momento estuvo constituido por la tabulación de las encuestas y se trabajó en el reconocimiento de las recurrencias y la saturación del dato, lo cual posibilitó aprehender el fenómeno estudiado de la forma más amplia. Luego un segundo momento estuvo constituido por el análisis y sistematización de los datos obtenidos en la entrevistas en profundidad, lo cual posibilitó re significar consideraciones hipotéticas formuladas a partir del análisis de las encuestas.

\section{Contexto de selección del muestreo}

El universo de recolección de la información con el cual se construyó la tesis, estuvo constituido por los estudiantes de Psicología, de la Facultad de 
Revista de la Escuela de Ciencias de la Educación, año 11, número 10, enero a diciembre de 2015. Páginas 303-312. ISSN 1851-6297. ISSN EN LINEA 2362-3349. LAS REPRESENTACIONES DE LOS ESTUDIANTES DE PSICOLOGÍA ACERCA DE LAS prÁcticas de enseñanza. Anahi Ayelen Marcovich

Ciencias de la Educación -Universidad Nacional del Comahue-, poniendo en foco y como informantes clave a la primer cohorte de graduados de la carrera; tesina que se inscribe en un estudio de caso.

El criterio para la selección de la muestra, estuvo delimitado por un lado a entender que al haber concluido con su formación en las asignaturas previstas en el plan de estudios, poseían una mirada de la totalidad de las prácticas de enseñanza de la carrera; por otra parte entendíamos que dicho recorte era lo suficientemente representativo de la diversidad constitutiva de la población más amplia de "estudiantes de la carrera de psicología". Diversidad que estuvo representada por diferentes categorías: clase, género, edad, etc.

Por otra parte, el recorte realizado en virtud de las prácticas de enseñanza estuvo acotado a los profesores a cargo de cátedras, excluyendo de dicha indagación a ayudantes de trabajos prácticos.

A los fines de reunir un grupo de graduados que permitiese la realización de las entrevistas, en un número no menor de tres, se esperó contar con un mínimo de nueve egresados, lo cual llevó tres fechas de colación.

Para la toma de la encuesta, el criterio que orientó la selección de la población se centró en identificar los estudiantes de la carrera que cursaban las últimas materias previstas el plan de estudios (es decir las correspondientes al $5^{\circ}$ año, $2^{\circ}$ cuatrimestre), como primer recorte; luego a partir de contar con la nómina de cursados se realizó una segunda selección que obedecía a identificar de esa totalidad aquellos estudiantes que, efectivamente, estaban cursando sus últimas materias es decir su último año. Esta tarea permitió circunscribir la labor de análisis a cuarenta y nueve encuestas.

En este punto tomó relevancia el desafío que consistió en aprehender el fenómeno estudiado de la forma más amplia posible. Por ello la propuesta de complementar la perspectiva de una investigación cualitativa con un enfoque cuantitativo no se trató de mezclar técnicas y métodos, sino de articular que cada instancia indagatoria sea pertinente para añadir profundidad al análisis. Dicho de otro modo, esta posición procuró enriquecer los análisis cualitativos basados en entrevistas semi estructuradas con el análisis cuantitativo de encuestas que nos sirvió de contexto.

\section{Construcción e Integración en categorías}

A partir de la saturación de los datos, se realizó un primer intento de integración en categorías, y teniendo en cuenta que la unidad de análisis del estudio realizado se centró en "las representaciones de los estudiantes de psicología (FaCE-UNCo) acerca de las prácticas de enseñanza", se formularon los siguientes constructos analíticos:

- Condiciones académicas

- Condiciones personales 
Revista de la Escuela de Ciencias de la Educación, año 11, número 10, enero a diciembre de 2015. Páginas 303-312. ISSN 1851-6297. ISSN EN LINEA 2362-3349. LAS REPRESENTACIONES DE LOS ESTUDIANTES DE PSICOLOGÍA ACERCA DE LAS pRÁcticas de enseñanza. Anahí Ayelen Marcovich

La construcción de estas categorías analíticas obligó a dar cuenta de qué entendemos cuando aludimos a ellas, explicitando el sentido de su uso.

La referencia a las condiciones alude al estado en que se encuentra un elemento en relación a otro, en este sentido se puede hablar de "buenas" o "malas" condiciones con respecto a una finalidad dada. También refiere a los "requisitos que establece un contrato" -en tanto dispositivo en uso en campo jurídico-, pero que en uso metafórico refiere a las condiciones de ingreso y tránsito de los estudiantes a una situación prescripta y reglada como la que ofrecen las cátedras universitarias. Se trata entonces, de un contrato tácito que enmarca derechos y obligaciones de ambas artes contractuales, compromiso, por otra parte que puede cumplirse o no.

Por otra parte el uso de académico, y si bien tiene varios sentidos el concepto, a los fines del trabajo de la tesis, acotó el sentido del mismo para denotar el uso habitual que específica un nivel de escolarización, y que en este estudio al que nos estamos refiriendo: es el universitario con su universo de funciones (docencia, investigación y extensión). Es decir como adjetivo refirió al conjunto de actividades que realiza el docente en educación superior. En función de lo dicho huelga decir que las condiciones académicas se materializan en una institución que es la Universidad, lo cual requiere tener presente la "especificidad" que la define, indica su dimensión pedagógico-didáctica que la enmarca y define su finalidad que es brindar enseñanza esperando producir aprendizajes.

Respecto del adjetivo "personal" ligado a las condiciones, se aglutinó/ agrupó aquellos indicadores que daban cuenta de la dimensión de lo subjetivo, enlazando las ponderaciones que realizaron los estudiantes referidas al intercambio, comunicación, y que en tanto prácticas humanas inscriptas socialmente recuperan su dimensión ética y emocional/ afectiva. La construcción de esta categoría nos invitó a entender las prácticas de enseñanza no como algo meramente instrumental sino como acciones que se guían por medio de valores éticos, propiciando la reflexión sobre la condición humana.

La indagación realizada permitió reconocer una representación mayoritaria respecto de las prácticas de enseñanza como "condiciones académicas", algunos indicadores que encontramos y que nos posibilitaron dar cuenta ella, fueron denominados como:

- Título: preponderancia valorativa del título de Psicólogo y la práctica profesional.

- Recorte y estructura: Conocimiento profundo de la materia, programa, del plan de estudios, articulaciones verticales y horizontales de la carrera.

- Figuras del oficio: alude a cierta organización discursiva que permite la comprensión, entendimiento.

- Ética: valoración respecto del respeto de los acuerdos implícitos -evaluación, coherencia, comunicación- y lo explícito. Coherencia entre teoría y práctica. 
Revista de la Escuela de Ciencias de la Educación, año 11, número 10, enero a diciembre de 2015. Páginas 303-312. ISSN 1851-6297. ISSN EN LINEA 2362-3349. LAS REPRESENTACIONES DE LOS ESTUDIANTES DE PSICOLOGÍA ACERCA DE LAS prÁcticas de enseñanza. Anahi Ayelen Marcovich

Se identificó una segunda representación acerca de las prácticas de enseñanza como "condiciones personales" que posibilitó condensar los aspectos ligados a lo afectivo/emocional. Los indicadores que posibilitaron su señalamiento:

- Posición de: estar disponible/dispuesto en la situación

- Pasión por enseñar: refiere a las ganas, el deseo, interés por enseñar

- El buen decir: elocuencia, formato discursivo que elabora el docente a los fines de la clase.

\section{Algunas conclusiones}

El trabajo de la tesina permitió reconocer y establecer una relación entre las representaciones de los estudiantes de la carrera de psicología, en diversos momentos, es decir al momento de elección de la carrera, al seleccionar la asistencia a dos teóricos por el interés de sus contenidos y en instancia de pensar su posible inserción profesional. El análisis del material empírico permitió establecer la preponderancia de una representación hegemónica, este último concepto a los fines de dar cuenta del dominio de una "entidad" por sobre otra.

Se pudo sostener la existencia de un imaginario colectivo ligado a las prácticas de enseñanza, en la formación del psicólogo, que señala una preponderancia en la valoración de que las mismas sean ejercidas por lo que se denominó "El psicólogo como enseñante".

En este punto y al interpelar acerca de las singularidades a la que remitía la construcción de tal categoría, fue necesario dar cuenta de una construcción que remite a dos campos disciplinares, es decir por un lado la formación de psicólogo que refiere a prácticas profesionales ajenas a la docencia, pero a su vez se inscribe en una posición de enseñante. Lo expuesto permitió abarcar aquellos rasgos que destacaron los encuestados y entrevistados como patrimonio específico de estos profesores. Así se pudo advertir la existencia de una valoración prioritaria que tiene que ver con un saber específico, sólo posible y considerado en términos de patrimonio de la formación del psicólogo.

En el análisis realizado oportunamente, los estudiantes señalan la necesidad y como requisito - para el ejercicio de la docencia en la carrera- de contar con el título de grado de psicólogo, acreditando en la medida de lo posible alguna titulación posterior de especialización en el área temática de incumbencia. Estas expresiones re-presentan y expresan un "orden social" entre diferentes saberes profesionales, que remite a un conocimiento compartido grupalmente y que constituye en sí lazo, en tanto identidad/identificación del continente "ser Psicólogo".

Esta ponderación especificó aún más, es decir señala ciertas prácticas del desempeño en el campo profesional por encima de otras, como lo es la práctica clínica. Los estudiantes permanentemente aludieron a la necesidad no 
Revista de la Escuela de Ciencias de la Educación, año 11, número 10, enero a diciembre de 2015. Páginas 303-312. ISSN 1851-6297. ISSN EN LINEA 2362-3349. LAS REPRESENTACIONES DE LOS ESTUDIANTES DE PSICOLOGÍA ACERCA DE LAS prácticas de enseñanza. Anahi Ayelen Marcovich

sólo de acreditar experiencia de campo, "consultorio", "tener calle", sino estar trabajando al momento del dictado de cátedra; lo cual implicaría no "tener" simplemente "la teoría en el bolsillo".

La posición de los estudiantes respecto de la experiencia, permitió entrever que de manera hegemónica el ejercicio de la profesión se "representa" como condición sine quo non para las prácticas de enseñanza en la carrera de psicología, y específicamente la práctica clínica, vinculando está última con la posibilidad de disponer de un mayor número de ejemplo capaces de sostener la construcción teórica del campo disciplinar. Representación que construye y orienta prácticas, en este sentido, dicha práctica se puede considerar como un facilitador de las prácticas de enseñanza para los profesores, sostenida por una posición de reconocimiento por parte de los estudiantes.

Asimismo la existencia de tal representación se puede presentar como un obstaculizador en términos de construcción de los contenidos de la cátedra, por un lado para aquellos docentes que no acrediten la práctica clínica (psicólogos o no), puesto que y en principio carecerían de ese reconocimiento particular que ponderan los estudiantes; pero por otro lado también para los estudiantes que pueden no encontrar valor al desarrollo de los teóricos per se, es decir una preponderancia de una posición negativa ante la situación y prácticas de enseñanza.

En este punto se interpretó que esta preponderancia del "Psicólogo como enseñante" se ve facilitada por la representación que opera como punto de partida en la elección de la carrera, pero a su vez este imaginario mayoritario se re-construye y consolida en el proceso de formación de la profesión, ello acontece en virtud de no sólo operarse la construcción de conocimientos disciplinares sino que verdaderamente se trata de un tiempo en el cual se constituye la identidad de la profesión. Una profesión con la carga de deseo, afecto y emoción que supone la elección en la formación de grado y el proyecto personal.

Lo expuesto permitió sostener que se opera un verdadero proceso de modelización de la profesión, la cual tiene como telón de fondo la práctica del psicólogo clínica y específicamente psicoanalítica, en la mayoría de los casos, pero que a su vez se presenta como lugar de llegada para inscribir la práctica profesional.

Es decir, más allá del recorrido realizado en el proceso de formación y que en la carrera de Psicología, de la Facultad de Ciencias de la Educación de la UNCo, opera realmente una apertura de aquellas representaciones originales que circunscribían el campo profesional al ámbito del consultorio y con el diván. Un recorrido que permite pensar sus prácticas como posibles en espacios/ terrenos que se articulan con las diferentes orientaciones que propone su plan de estudio, pero que en principio y cual se entiende en este estudio no alcanzan para modificar la orientación hegemónica de sus prácticas cuando piensan en su futuro profesional. En este sentido, aparece como recurrente pensar en la 
Revista de la Escuela de Ciencias de la Educación, año 11, número 10, enero a diciembre de 2015. PÁginas 303-312. ISSN 1851-6297. ISSN EN LINEA 2362-3349. LAS REPRESENTACIONES DE LOS ESTUDIANTES DE PSICOLOGÍA ACERCA DE LAS prácticas de enseñanza. Anahi Ayelen Marcovich

práctica profesional en consultorio y clínica, luego de construir la experiencia (tutelada) necesaria que pueda dar cuenta "de los cuidados que requiere el otro".

Lo expuesto adquiere pleno sentido al considerar la finalidad que cumple la representación social, opera como posibilidad de transformar lo desconocido, es decir la práctica profesional en sí y a partir de la graduación, en algo familiar. En este punto, entonces, podemos pensar que tal representación hegemónica aparece como un horizonte de significado en el cual construir el desempeño profesional en "algo conocido", en virtud de la falta de certezas respecto de cómo sus primeras prácticas profesionales vaya incidiendo en estas representaciones y en la modelización de la práctica in situ.

La indagación realizada respecto de las representaciones sociales acerca de las prácticas de enseñanza posibilitó reconocer, entender, describir y explicar alguna de ellas; permitiendo entrever un recorte del conocimiento que subyace a la construcción de conocimiento en las prácticas de enseñanza de la carrera de Psicología.

En este sentido, su elaboración lejos de presentarse como acabada supone una construcción provisoria, que conduce a nuevos interrogantes que se vinculan con las prácticas de enseñanza en sí, y en el marco de pensar la mejora constante de nuestras instituciones educativas. Por ello es lícito interrogarse acerca de cómo son las buenas prácticas de enseñanza que reconocen los estudiantes, no ya y a partir de las voces de los mismos, sino y a partir del ejercicio del "psicólogo como enseñante", es decir qué hacen los psicólogos para enseñar en tanto buenas prácticas.

¿Se puede pensar, por otra parte, que la representación del campo profesional hegemónica: consultorio y clínica, es re-construida dialécticamente, por prácticas de enseñanza sostenidas por las representaciones de los psicólogos enseñantes? En el sentido de práctica valoradas "entre" el cuerpo de profesores.

Asimismo quedan incógnitas respecto del psicólogo en la posición de profesor y cómo vincula "el saber didáctico" -entendiendo por ello lo que implica específicamente la labor docente- en el contexto de la enseñanza de psicología y atendiendo a la complejidad de la diversidad de objetos de estudio que presenta.

En consonancia con lo expuesto surge el interés también en pensar, cómo resuelven la enseñanza aquellos docentes-psicólogos o no, que no poseen experiencia clínica que permita "ejemplificar", o acaso el requerimiento del uso de la experiencia sólo tiene en foco "determinadas" cátedras por sobre otras menos valoradas en la formación profesional más general.

A modo de cierre se puede destacar que con la realización de la presente tesina se pudo arribar a ciertas respuestas respecto de la problemática indagada como así también atender a los interrogantes que guiaron la indagación, posibilitando la construcción de significaciones de eso que pretendía conocer. 
Revista de la Escuela de Ciencias de la Educación, año 11, número 10, enero a diciembre de 2015. Páginas $303-312$. ISSN 1851-6297. ISSN EN LINEA 2362-3349. LAS REPRESENTACIONES DE LOS ESTUDIANTES DE PSICOLOGIA ACERCA DE LAS prácticas de enseñanza. Anahí Ayelen Marcovich

\section{Notas Bibliográficas}

(1) Cabe destacar que dicha carrera fue aprobada por la Ordenanza $N^{\circ} 0434 / 03$ y modificatorias $N^{\circ} 596 / 04$ y 0224/09, del Consejo Superior de la Universidad Nacional del Comahue. Es decir, mi participación como docente de esa casa de altos estudios, se inicia con la primera cohorte de cursantes en la carrera, que contó con 1124 inscriptos.

\section{Referencias Bibliográficas}

- $\quad$ Cattaneo, M. E., \& Ruíz, A. R. (2007). Motivación y rendimiento académico: ¿es importante el ciclo introductorio en la disciplina? IV Congreso Nacional y II Internacional de Investigación educativa. ISBN 978-987-604- 035-8

- D'Ancona, C. (1999). La metodología cuantitativa: estrategias y técnicas de Investigación social. Madrid: Síntesis.

- De Pascuale, R. (2004). Los própositos de la enseñanza y la intervención didáctica.

- Jodelet, D. (1986). “La representación social: fenómeno, concepto y teoría”. En S. Moscovici: Psicología Social II. Barcelona: Paidós.

- Litwin, E. (1996). "El campo de la didáctica: la búsqueda de una nueva agenda". En A. W. de Camillioni, M. C. Davini, G. Edelstein, E. Litwin, M. Souto, \& S. Barco: Corrientes Didácticas Contemporáneas. Buenos Aires: Paidós.

- Litwin, E. (1997). Las configuraciones didácticas. Buenos Aires: Paidós Educador.

- Moscovici, S. (1979). Psicoanálisis, su imagen y su público. Buenos Aires: El Huemul.

- Moscovici, S. (1986). Psicología Social I y II. Barcelona: Paidós. 